\title{
APPLICATION OF EPC STANDARDS AND MOBILE NETWORKS SERVICES TO ENHANCE THE QUALITY OF POSTAL SERVICE
}

\author{
Juraj Vaculikk, Jiří Tengler ${ }^{2}$, Ondrej Maslák ${ }^{3}$ \\ ${ }^{I}$ University of Žilina \\ Žilina, Slovak republic, Univerzitná 1, 01026 \\ Phone:+421-41-513-3132,juvac@fpedas.uniza.sk \\ ${ }^{2}$ University of Žilina \\ Žilina, Slovak republic, Univerzitná 1, 01026 \\ Phone:+421-41-513-3121, jiri.tengler@fpedas.uniza.sk \\ ${ }^{3}$ University of Žilina \\ Žilina, Slovak republic, Univerzitná 1, 01026 \\ Phone:+421-41-513-3145, maslak@fpedas.uniza.sk
}

\begin{abstract}
This article deals with new technologies and methods to optimize the transport and delivery of postal mails. Introduction of this article defines the technologies and procedures that postal operators have started to use. The main part of the article describes specific implemented solutions of several postal operators and their benefits. The last part shows using of EPC standards in a processes chain. The conclusion of the article is devoted to the evaluation of these technologies and their potential in the area of postal sector.
\end{abstract}

Keywords: delivery mails, mobile technology, post, RFID technology, EPC standards

\section{Introduction}

Information, currently, is still the best competitive tool and those who get control of it first, gain a big advantage. Using automatic identification technology can extract information from manufacturing, logistics and business areas in near real time. These technologies are indispensable aid in identifying, tracing and tracking of any object. Recent advances in RFID will enable us to allow, in the future years, to obtain any object in heterogeneous environments as a small RFID device. This device will be able to monitor, control and ultimately may have an important role in a number of key decision-making processes. Therefore, RFID technology can be described as very beneficial, as it is currently implemented in more areas than it might initially seem. But there are other technologies that have experienced tumultuous development besides RFID technology, namely the mobile telecommunications. This technology has been gradual convergence of telecommunications with information technology and new media course (together mobile telecommunications) and contributed to a massive change in orientation, not only in industry but also in one's daily life (Fuchikova, Kebo 2012).

Mobile telecommunications technology has played an important role in accelerating and moving into the areas of communication and processes, which are important in the terms of time. It is these two contactless technologies that we are currently considering as the key ones, since they have made the greatest enrich and simplify the existing processes in all areas of human activities. It can be said that both are beneficial only in specific industrial areas, which means that one technology brings benefits to those processes and areas where it would be unrealistic to use the other and vice versa. It is natural to assume that reaching the desired track record (enrichment) could be achieved just by integrating these technologies mentioned above, and this would also eliminate the unreality of the use of technology in inappropriate areas. The combination of advantages of both technologies could of course not only reach new markets, but also improve processes in the existing industrial sectors.

\section{Mobile telecommunication in postal sector}

One of the areas, where mobile telecommunications have found their place is just the postal sector. This technology makes it possible to move the communication to the end customer. It may be important especially in the delivery of postal mails, because mail recipient is not usually aware of the arrival of mail 
to his address. Precisely in this area, there is the possibility of streamlining this process through mobile telecommunications, because it allows communication with the end customer (recipient of postal mail) (Bolarin 2011). No less important is the communication with the sender of postal mail that could be informed of the delivery of mail through mobile telecommunications.

\section{Analysis of the use of mobile communication postal operators to streamline postal delivery process}

At present, a large number of postal operators are using mobile telecommunication to streamline delivery processes. There are many solutions how to use this technology to improve the above-mentioned processes, but all they have common ideas. These ideas are:

- $\quad$ provide information about delivery status of mail to the recipient postal mail,

- provide information about delivery status of mail to the sender postal mail,

- $\quad$ arrange a day, time and place of delivery postal mail to the recipient.

Research has found that most postal operators use mobile services for the following activities (services): telephone conversation with the recipient of postal mail, for sending an SMS message to the recipient of postal mail, for sending an SMS message to the sender of postal mail, for sending an interactive SMS message to the recipient of postal mail or for sending an SMS message to the recipient of postal mail, after delivery of postal mail into postal box; with the standard forms of communication:

- Telephone conversation (notice) - It's a service for communication with the recipient of postal mail. This communication provides information on status mail or place and time of delivery of postal mail.

- SMS message to recipient - It's a service that provides information about status and the planned delivery of postal mail via SMS message to mobile telephone recipient of postal mail

- SMS message to sender - It's a service that provides information about the delivery status of postal mail to the sender of the postal mail. SMS message usually sent after the delivery of postal mail.

- Interactive SMS to recipient - This is a special type of service that is based on two-way communication. There is mean SMS communication between postal operator and recipient of postal mail. The recipient will receive SMS message, and the first part of message content preliminary information on delivery of postal mail to the recipient address. This part is the same as in the two previous cases. The second part of SMS message contains information about the possibility of changing the date of delivery.

In the second part of SMS message there are predefined alternate days for delivery. Each day is represented by a numerical choice. If the recipient of postal mail wants to deliver the postal mail on a different day, he sends an SMS with an appropriate answer with a numeric choice, which is predefined in the incoming SMS message.

SMS message to recipient after delivery - This is again a specific type of services. In this case, the SMS is sent to the recipient after delivery of postal mail into postal box.

A very specific and new way to use the SMS service in postal sector is through automated postal dispensaries (the so-called Pack Boxes or Pack Station). After every transport and storage of postal mail there is generated and sent an SMS to the recipient with information on storage mail. The SMS message contains info about location and designation of pack station and access cote to open the compartment. The use service of mobile network can result in:

- attractiveness of postal services,

- reduction or elimination of the cost of the failed delivery of postal mail,

- replacement of paper notification of delivery failure postal mail,

- possibility for the recipients to participate in the final phase of the process of mail delivery,

- $\quad$ streamline access of mail recipients.

\section{Other possibilities of mobile telecommunications to improve the process of delivery postal mail}

\subsection{Sending SMS confirmation of receipt mail to the sender and monitoring postman}

The principle of this solution lies in the arrival of the postman to the address of the addressee and probing barcode Smartphone. The value of this code would be read out and recorded in the database. On the basis of this initiative there would be generated and sent SMS messages to the sender mail. The 
process of the solution would be undertaken in real time. The content of the report will inform the delivery of the parcel in time and space.

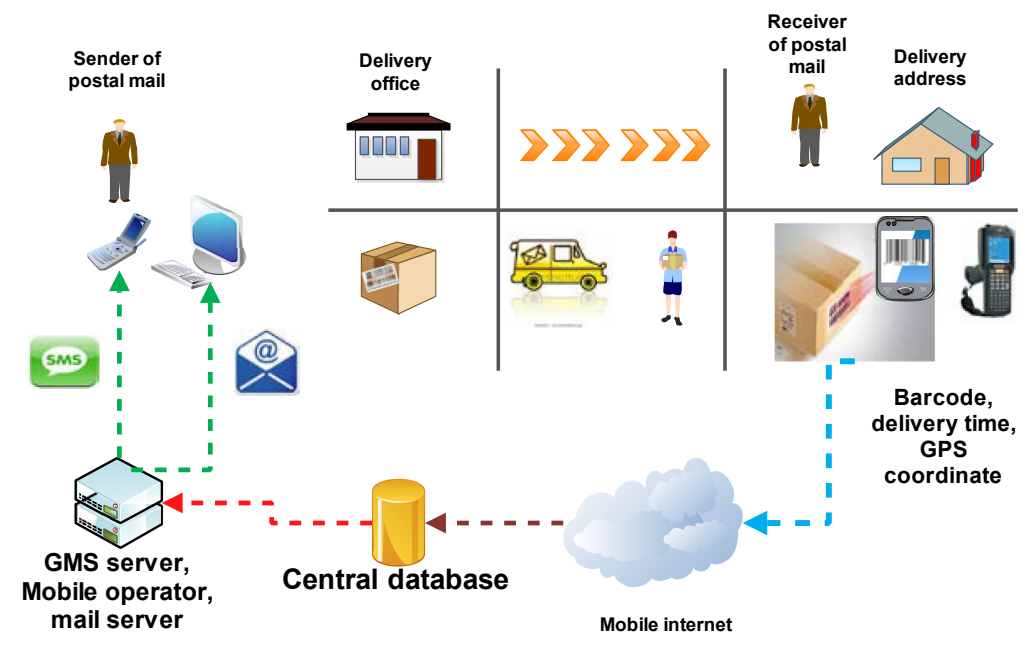

Figure 1. Principle of this solution 1

\subsection{Delivery of postal mail to the selected GPS}

The principle of this solution lies in the additional delivery of mail to the GPS coordinates, the recipient who sent the mail through SMS. GPS address by means of a smart phone. Based on this GPS coordinates, postal operator delivers, for a fee, mail to the place designated addressee mailings. All communication take place by means of the SMS messages sent.

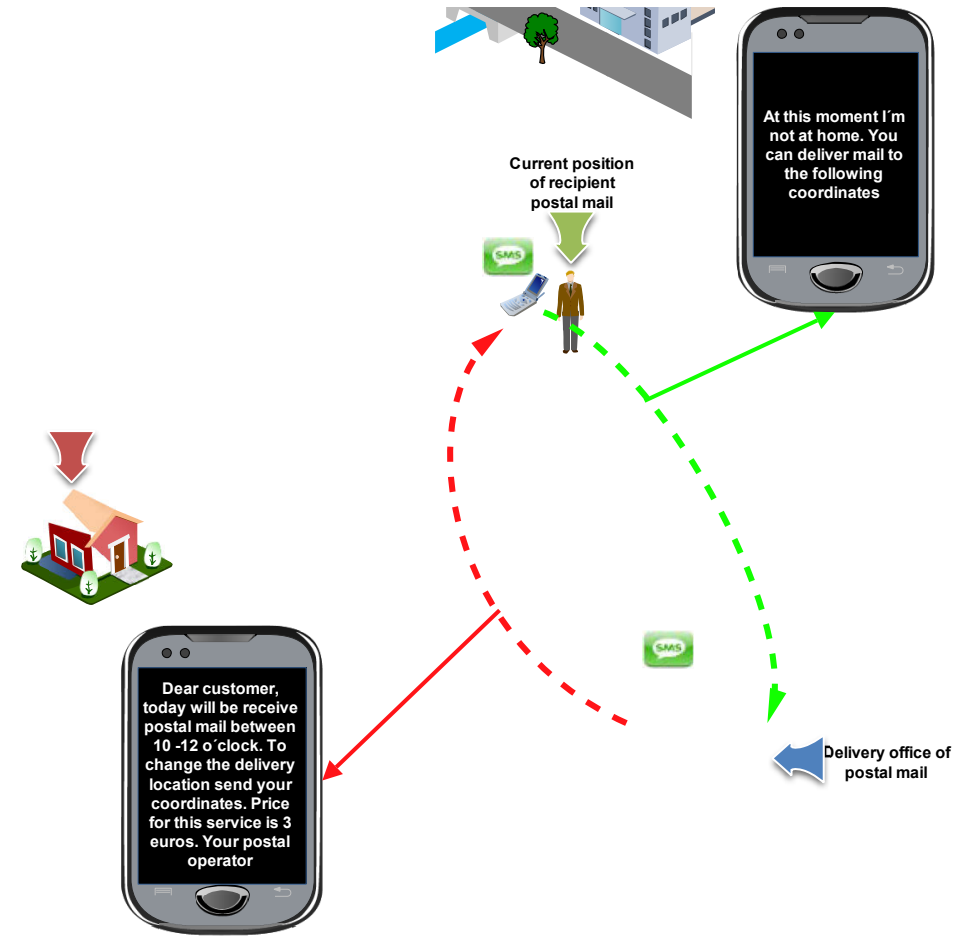

Figure 2. Principle of this solution 1

\subsection{Draft model to improve the process of mail delivery}

Given the nature and scope of these two technologies, it is evident that each provides a positive effect in different planes within the shipping process. Let us then outline what the main effects of these technologies bring in terms of the process of monitoring the transport and delivery (delivery) of mail. 
RFID technology is a complex, combining a number of different computing and communications technologies to achieve the desired objectives. (Kolarovszki, Dúbravka 2010) RFID technology enables real-time consignments bearing RFID identifier uniquely specify the exact time and location specific mailings at various stages of the production process, that enables to provide valuable information about the input or output of the consignment under such processing centres. Generally, it is an element of automation, which provides information without human intervention.

Mobile services in turn allow for certain conditions to facilitate communication between the postal operator and customer, using their services similarly, as mentioned in assessment using the services of mobile networks in the postal sector. The whole essence of the proposal is based on simplicity and eliminates the need of human labour to optimize mail supply (delivery).

Before starting the planning and designing the process model we define some basic principles that should be met in the development of the proposed model:

- eliminate the need of human intervention (necessary labour) under the proposed model,

- the lowest level necessary hardware and software,

- $\quad$ automate the entire process of the proposed model,

- allow the sender to change the mail delivery available,

- $\quad$ simplicity and complexity of the system,

- pôsșibility to use additional hardware and software,

- $\quad$ applicability of primarily recommended insured letters and parcels.

Therefore, the way to integrate these technologies within the proposed model has been more noticeable, trying to describe the scope of each of these technologies on a general model of the transport and delivery of postal items. Out of the above, the two technologies have been used in the design as well as some other additional technologies, which to a certain extent, within the model, eliminates the need of a higher number of necessary hardware. This additional element is just bar code technology.

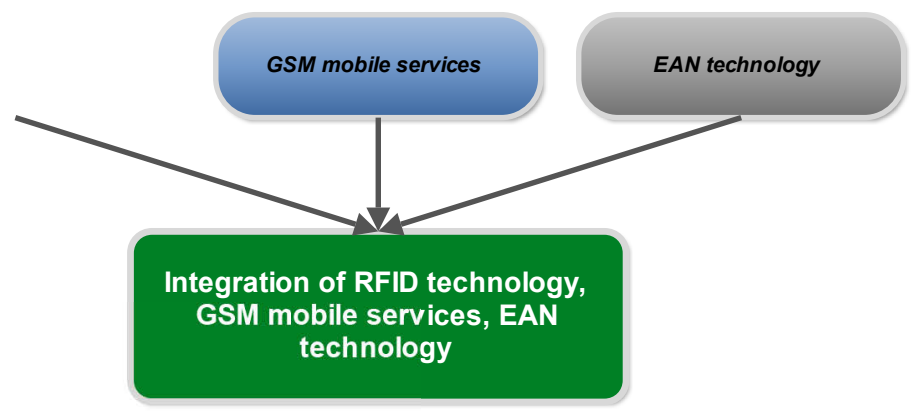

Figure 3. Integration technologies

Before we begin to define the areas of use of these technologies in the postal sector, it is necessary to outline a simplified general model of transport and delivery of postal items. In view of the relatively high cost of design and, generally large volumes of mail distributed to the light of the principles, we deal only with recommended, insured letter-post and parcel shipments.

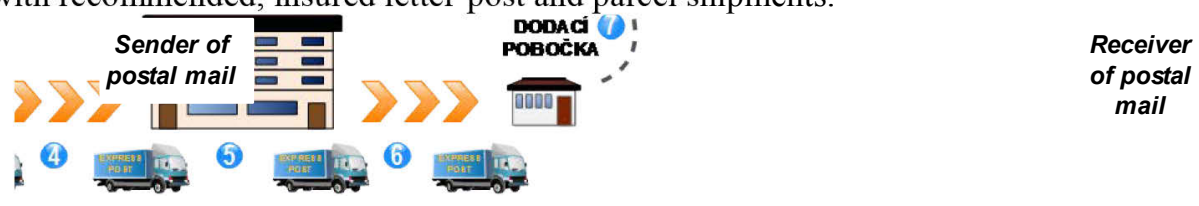

Input central processing center
Output central processing center

Post
office

Delivery

office

Figure 4. Principle transport in postal transport network (Vaculik, Zelik 2010) 
Simplified principle of transport and delivery of postal items is implemented in the following points:

1) The consignor comes to posting place and makes recommendations, insured letter or parcel,

2) The consignments are transported to the input of the central processing centre

3) are processed and categorized according to the address of lines of output to the central processing centres,

4) The consignments are transported from the central processing centre input to output processing centre

5) again leads to sorting of mail by individual delivery points,

6) as follows, categorized shipments are transported to various delivery points,

7) Then, shipments are delivered to the addressees.

We can now consider the applicability of technologies due to the simplified general model of transport and delivery of postal items by the principles set out in the design of the model. The proposal is to construct a model that would allow monitoring the consignment in the postal mail transmission network operator, and arranged at certain points of communication with the addressee of the handling and disposition of the consignment. As for the nature of the design model, we can conclude that the function will perform at two levels, namely in:

- identification part and

- communication part.

By the identification of the shipmen, it will be tracked from submission to delivery through passive identification technology to posting and delivery locations, and also through active identification technology in the processing centres. By passive identification technology we mean the aforementioned barcode technology and by active identification technology - the RFID technology.

Names of passive and active identification technologies have been chosen because of the need of separation technology, which is needed to identify mail within individual branches i.e. postal operator places human involvement, and those without the human factor bypass. The essence of communication is the ability to convey one-sided or two-way communication between the operator and the addressee of the postal mail.

When selecting appropriate options for the proposed model, they can be derived from the length information stored in the RFID identifier, i.e. the number of characters that are stored in memory. This value is usually quite high and if it's printed in bar code would thus long value obstructing the possibility of manual data entry, assuming no bar code reading device for storage in the database. This is one of the reasons why we decided for the second option, there will be a relationship between the information stored in the barcode and the information stored in the RFID identifier in the smart label. So there will be predefined table relationships between the data from the barcode and the data from RFID identifiers. When you enter barcode into the database of the deposit site is still attaches the data from RFID identifier stored in the predefined table sessions. The principle is shown in the following figure (Tengler, 2013).

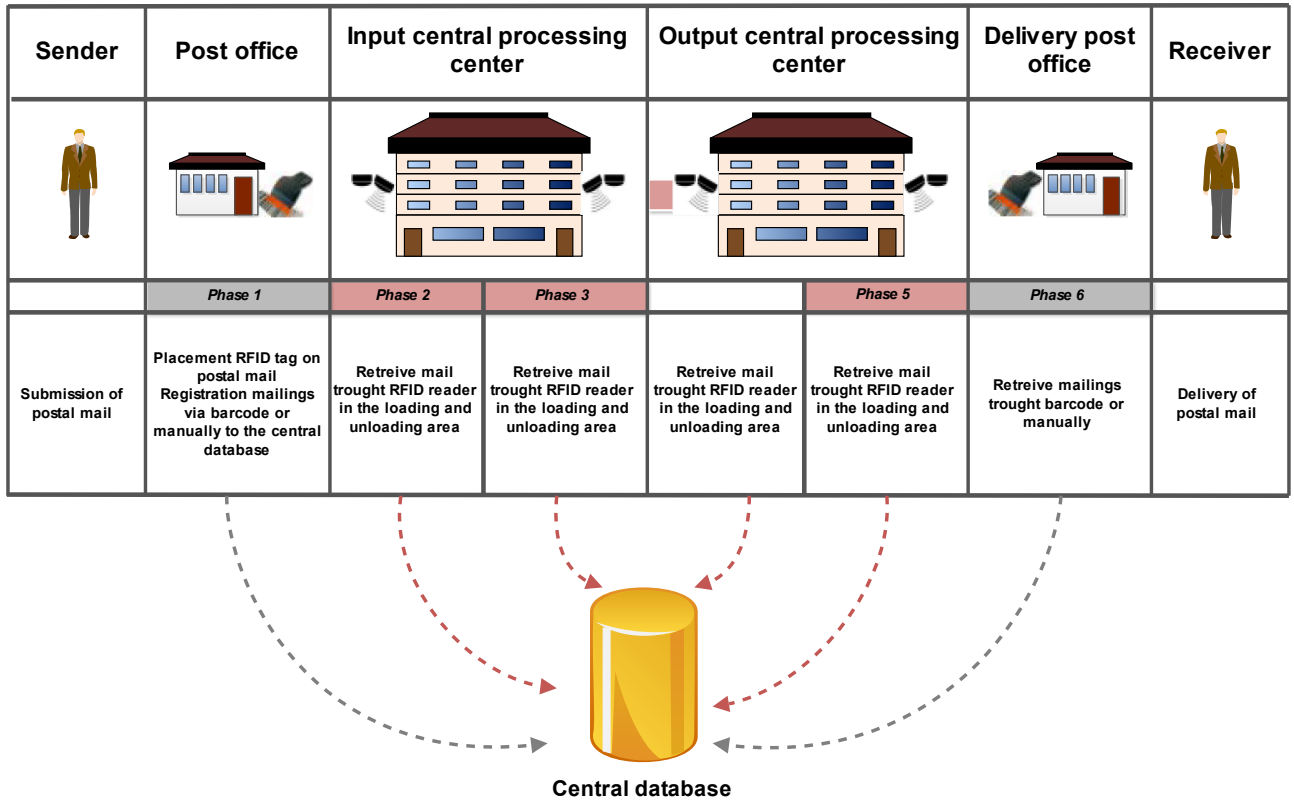

Figure 5. Principle of this model (Tengler 2013) 


\section{Anticipated benefits of the proposed system}

As for the based on the model design system, we can say what benefits might accrue to all participants in the process of administration, transport and delivery of postal items, the sender, addressee and postal operator. Taking benefits for postal operator may be primary or secondary.

\section{Benefits for the customer (shipper)}

- $\quad$ detect the condition, respectively, delivery of mail via SMS or e-mail,

- increase comfort services for the sender's mail.

Benefits for the customer (the addressee)

- increase comfort services for the addressee,

- improved accessibility to postal consignment to the consignee,

- obtain information about the planned delivery of mailings, before the completion of the transport of the consignment to the place of the delivery postal operator,

- option available to change the shipment, i.e. to change the date of delivery of the consignment, respectively the shipment to the delivery location postal operator via SMS or email.

\section{Benefits for the postal operator}

\section{The primary benefits}

- The flow of mail,

- monitoring compliance with quality requirements for the provision of postal services,

- the possibility of reducing costs in relation to the identification and elimination of bottlenecks in the distribution network,

- the possibility of cost reduction due to the reduction or elimination of unsuccessful delivery attempt and also in connection with the issuance of the consignment at the place of delivery postal operator in an unsuccessful delivery attempt,

- Possibility to reduce the percentage of non-delivery of mail at the first attempt.

Secondary benefits

- Using subsequently implemented infrastructure for monitoring transport units (postal cages, containers, crates, bags and other) under their postal operator of the transmission network (Tengler, 2013).

\section{Creation of real models}

A custom application was created in an environment middleware OnId/AMP, database system based on MySQL and Ozeki SMS Server. Functional diagram consists of two main interlinked parts. The first part (Fig. 11) consisting of processors FilterEqualTo, EarlyDecoupler, InsertProcesor and InlineSelectProcessor provide input of relevant information during the process of each processing centre.

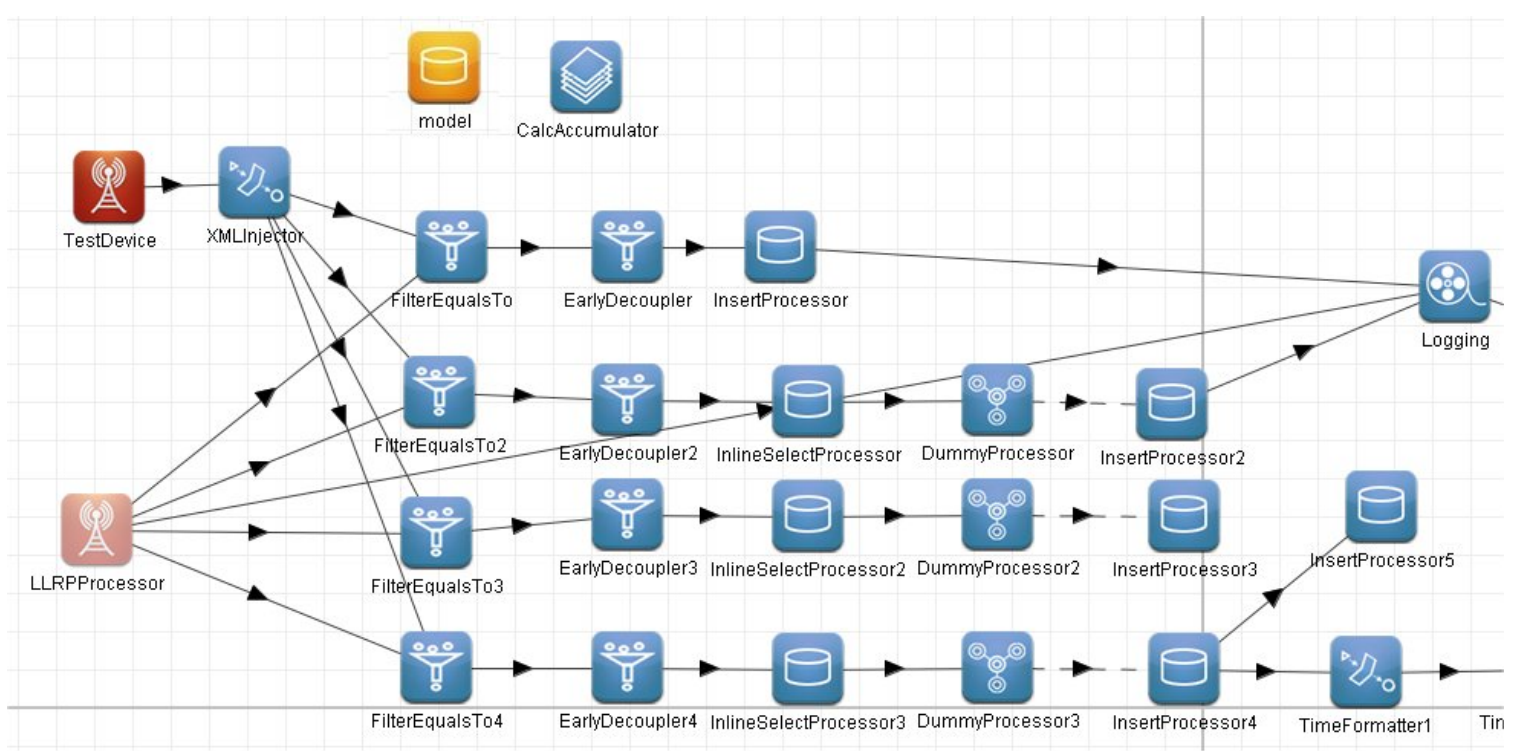

Figure 6. First part of real model 
The system contains several mutually cooperating tables in the database "Model", in which the individual has entered manually input, during processing chains and processing SMS and its notification.

The last part of the model (fig. 7) consists of processors for the preparation of a short message (TimeFormater, QueryExecutor, MessageTransformer) and its transfer to Ozeki SMS Server to manage its transmission through the mobile network operator and processing feedback notification (InlineSelectProcessor and SMSSender).

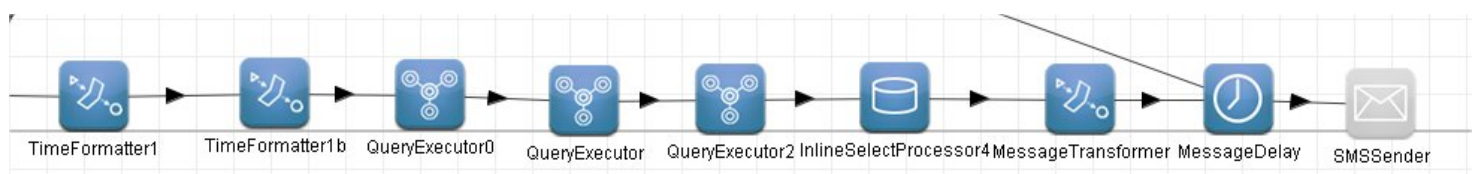

Figure 7. Second part of real model

\section{Use of EPC codes, created by the existing EPC standards in mail and logistics}

As it has been shown in Fig. 8, throughout the processing chain is involved in three basic parts RFID, GSM mobile technology and EAN respective EPC standards. In the previous sections we have considered the particular technology of RFID and mobile technology. But to the entire communication chain must be able to work some elements of standardization. Therefore, the final parts look more like the EPC standards and their application in the processing chain.

Implementation of RFID - EPC tag second generation (EPC GEN2 tag) to the postal service would be an increase in efficiency and productivity of the sector. Price tags are steadily declining and, therefore, the postal sector should pay more attention to the integration of this technology into their business processes, thereby ensuring not only increases of efficiency and productivity, but also increase of the visibility and accuracy of postal operations.

Standards represent the cumulative inter-conditional actions and measures that lead to efficient unification recurring solutions or can be seen as determining the performance requirements of raw materials and products, the manufacturing processes, to achieve uniform quality and functional use.

EPC codes, created on the basis of the existing standards may be used throughout the supplydemand chain, from producer to consumer. Mail can also be used with such EPC codes in their activity. The proposal to use the developed EPC code, based on the existing standards, is described in the following example (see Figure 8).

Imagine postal processes such as Supply Chain. The automatic data collection throughout the chain will serve data carrier, in which the memory will be stored the stored PC code itself, in this case, the EPC Gen2 RFID tags. We have to identify Gen2 EPC RFID tags

EPC scheme used in this case:

- $\quad$ SGLN to uniquely identify physical locations, specific place,

- $\quad$ SGTIN to uniquely identify a specific type of product,

- $\quad$ SSCC to uniquely identify logistic units. 


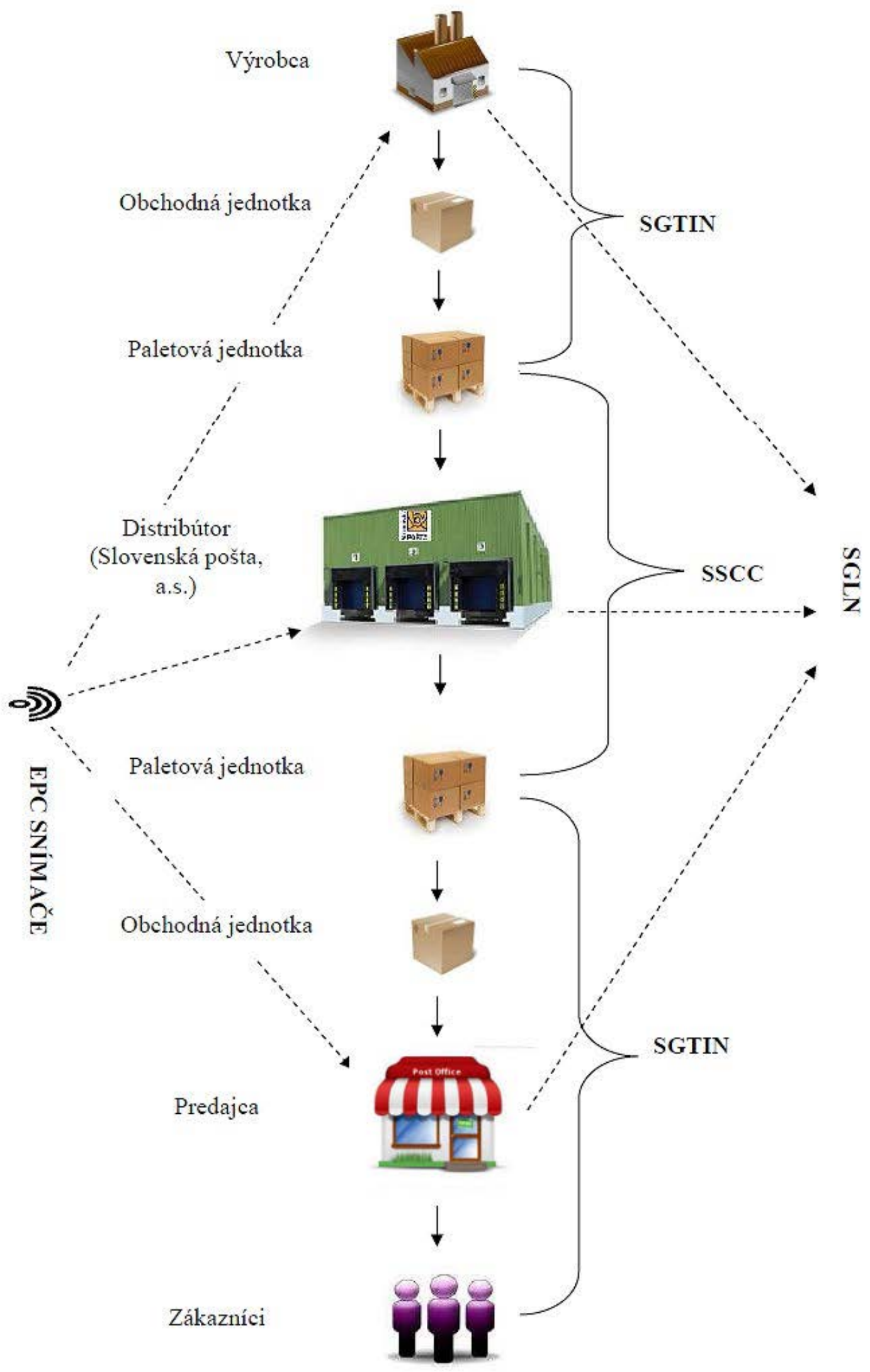

Figure 8. Use of EPC codes in mail and logistics 
The data encoded in the tag memory will be scanned EPC sensors. EPC sensor will be placed at strategic points in the supply chains in order to track the movement of goods in the chain. Sensor data then executes software EPC middleware, which sends the data to the EPC IS or other existing enterprise information systems. Sharing data provide EPC IS, from which the distributor can (eg Slovak Post) require specific information about the product. Search Services (Discovery Services) allow users to access data related to a specific EPC code, and also allow users to request access to this data.

Therefore it has been necessary to add to the whole processing chain use the EPC standards. An essential part of their use is to understand the transformation of information between the imposition of the carrier identifier (RFID tag) and useable information hierarchically higher system (ERP).

\subsection{Forms of EPC codes}

EPC URI (uniform resource identifier) is the preferred way to uniquely identify specific physical object within information systems. EPC URI is a string that shall take the following form:

urn: epc: id: scheme: component1.component2....

Where the scheme is an EPC scheme and component1 and the following sections represent the elements used by GS1 key corresponding to the EPC code. Example for SGTIN:

urn: epc: id: SGTIN: GS1firm_prefix.kind_of_goods,serial_number ->

urn: epc: id: SGTIN: 0614141.112345 .487 [13]

The structure of the EPC URI guarantees the uniqueness of all physical objects and applications around the world. EPC codes may take three forms. In computer systems, including electronic documents, databases and electronic messages shall have EPC code form. Another form, which may EPC codes acquire, is the form of the so-called EPC tag URI. Binary data structures defined in the Tag Data Standard are intended for use in RFID Tags, particularly in UHF Class 1 Gen 2 Tags. Specifically, it specifies that memory in these tags consists of four separately addressable banks, numbered 00, 01, 10, and 11. Bank 00 consists of access and kill password, bank 01 contains its own EPC, bank 10 is defined by the manufacturer and finally bank 11 is available to the user.

Memory bank code EPC RFID tag second generation also includes EPC code and "control" information (filter), which is used to guide the process of collecting data from RFID tags. This information can be used to filter tags, or to improve their reading efficiency. EPC tag URI is a URI string that represents a specific EPC code with specific settings for the "control" information stored in the memory bank EPC code. In other words it is a text equivalent of the entire contents of the EPC memory bank. This form is often used by the data collection, when reading RFID tag of "control" information interest application that provides data collection.

The EPC global Architecture Framework is used in the form of "events at the application level." Examples EPC tag URI when used SGTIN key:

urn: epc: tag: SGTIN-96: Filter.GS1_firm_prefix.kinf_og_itens.serial_number ->

urn: epc: tag: SGTIN-96: 3.0614141.112345.487

Another form, which can acquire EPC codes, is a form of binary encoding. Memory bank code EPC RFID tag contains the second generation compressed encoding EPC code and "control" information in a compact binary form. Converting between EPC Tag URI and binary form in the second generation RFID tag is 1:1. Binary form is used for low level software or hardware and usually converted into the EPC Tag URI or URI clear identity before being presented to form EPC code application logic. While pure identity URI is independent of RFID, EPC Tag URI and binary encoding are specific to the second generation of RFID tags (RFID EPC Gen2), because they contain a unique EPC identifier in addition to the "control" information. The EPCglobal Architecture Framework, this form uses the "Protocol for Sensor low level" and the "Air Interface Class 1 Gen2 and High Frequency".

The best way to show the transformation of information illustrates using the Smart Label, which is a combination of RFID technology and standard bar code in a single carrier (Fig. 9) 


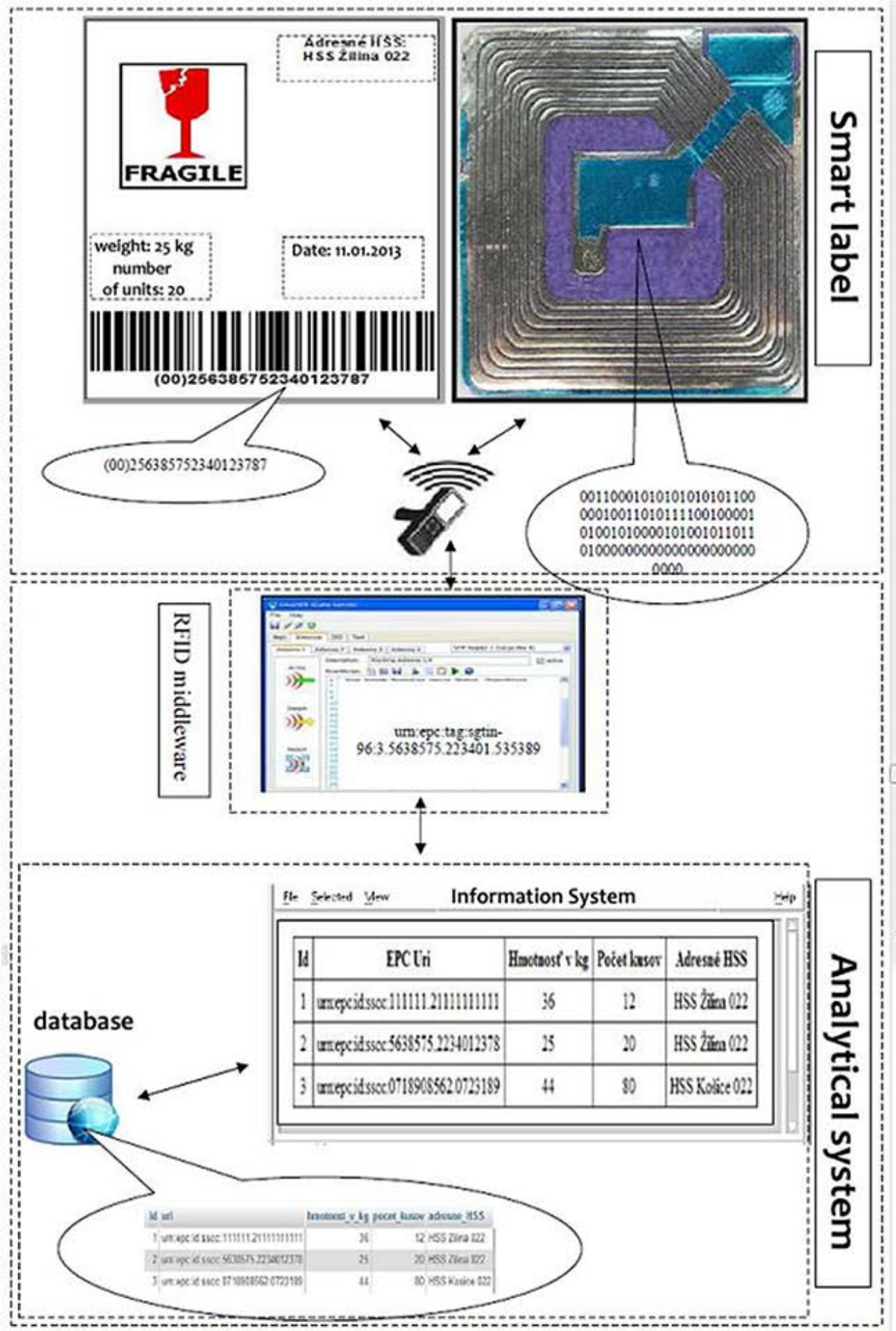

Figure 9. Use of EPC codes in the mail and logistics

Since we use middleware which does not directly support the EPC standards, it was necessary to provide management of their own means. As we can see in figure 10 for the decoding verification there was created a simple application in OnID/AMP middleware environment too. 


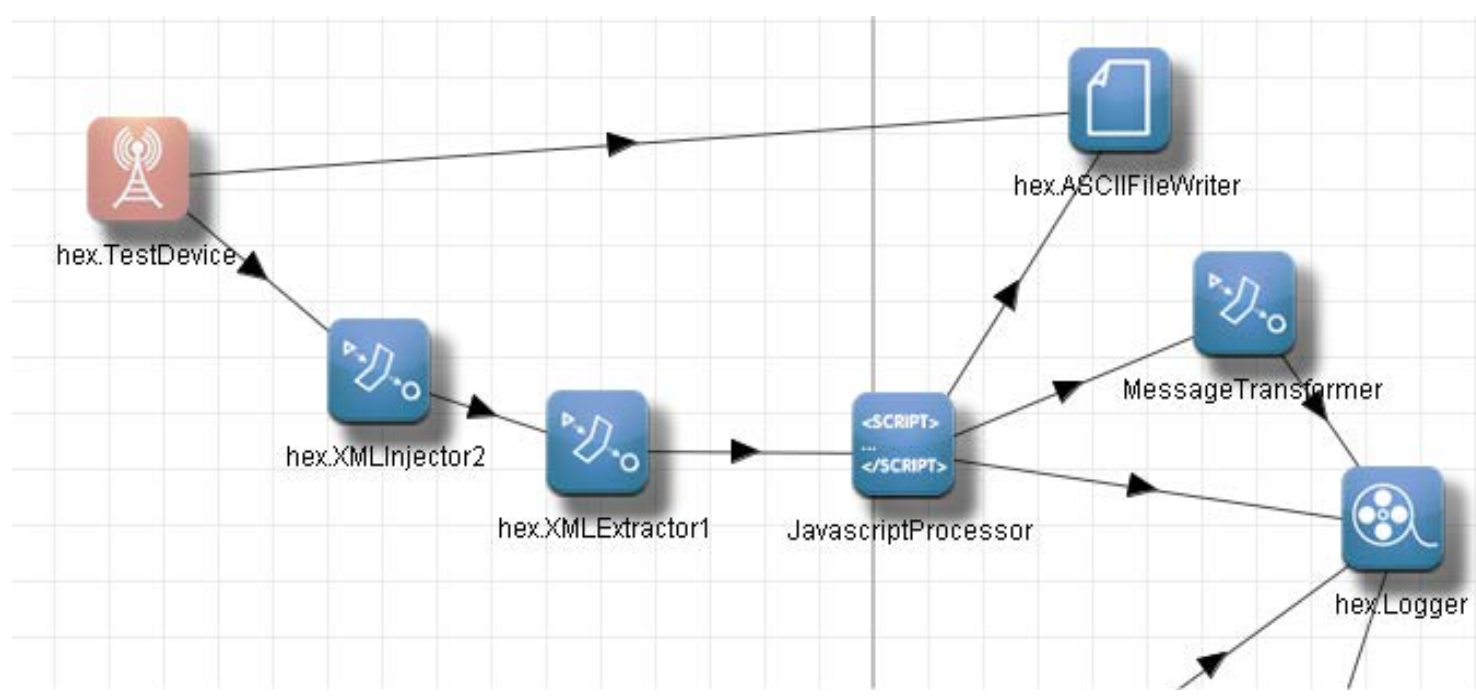

Figure 10. Procedure for decoding the content of identifier

At the entrance gate (hex.TestDevice in fig. 10) the identifier is read. Standard representation is in the hexadecimal form in the xpathe /reading/tagId. For example:

\section{$3140677 A$ F407 1C2B 67000000}

The next step is to adjust this form by hex.XMLInjector processor and XML.extractor processor. The adjusted form will be suitable for custom processing in a JavaScript - processor JavascriptProcessor in the scheme above. The first task of the processor is to save incoming massage to the variable content that is executed by this code:

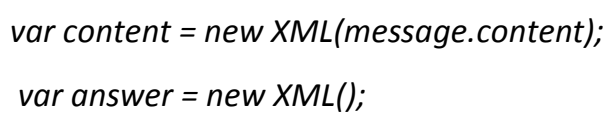

Next task is transformation of hexadecimal format to binary format for example by $\mathrm{H} 2 \mathrm{~B}$ (Hex to Bin) function, which may look like this:

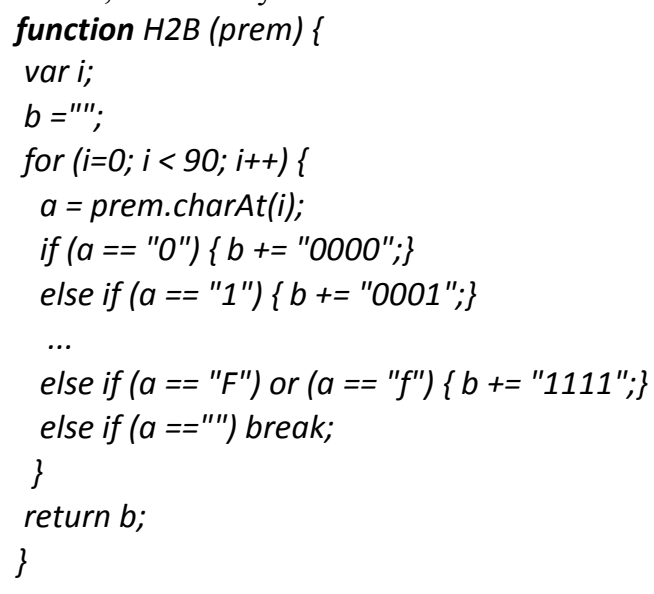

Result of function is in binary form:

$<m s g>$

001100010100000001100111011110101111010000000111 000111000010101101100111000000000000000000000000 $</ m s g>$

In further processing, the binary code is transformed according to the EPC specification (partition, filter) for each header and we can get individual parts of EPC structure in the XML report: 


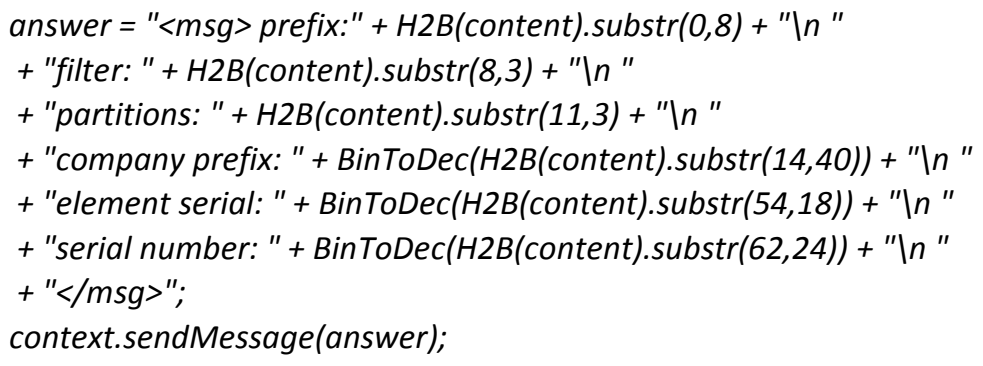

By using the sendMessage method there is generated an output message in the form:

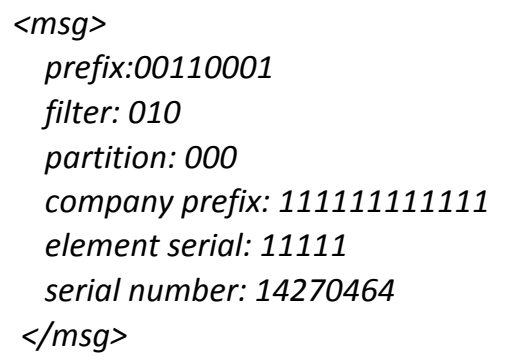

Next, the final format of EPC standard is created. This format is suitable for entry into the EPCIS system and by methods of sendMessage requested information is sent for further processing and by using the method outLine we can send information to the log system.

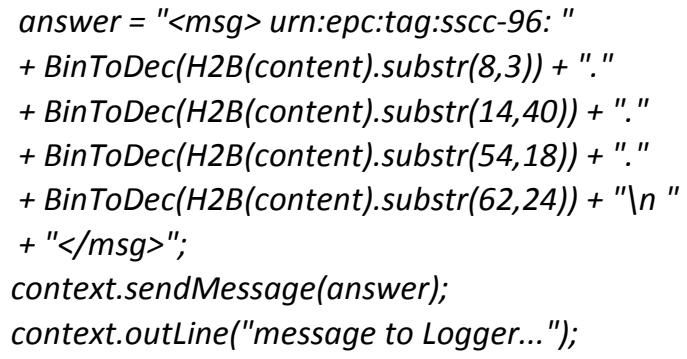

The resulting report is in the XML format:

<msg> urn:epc:tag:sscc-96: 2.111111111111.11111.14270464 </msg>

The description is used to decode the identifier for containers: SSCC-96 bits length. In a similar way, it is possible to create a number of processors to decode any input data according to the standard of an identifier of the EPC and its transformation from binary format into a form accepted in the further processing in the form of urn, uri or pure identity regardless of encoding of the identifier and the bit size of the stored information (8-bit, 6-bit, etc.).

In the same way, for example, there can be decoded manufacturing information of any tag (TID Transponder Identification), where we can know by the encoded data about the used chip, memory size, type and serial number or user memory. By using Application Identifier (AI) we can store in the user memory further information: the length of goods, expiration date, and many others according to specifications and standards of GS1.

This makes it possible to link any postal, transport or logistics chain system working under EPC standards and RFID technology. We can label not only the shipments themselves within delivery chain, but also the transport of containers, pallets, vehicles and processing centres including end-Post offices buildings.

Manufacturer (postal operator) produces a business unit (letter, parcel). To indicate the business unit, we use EPC code developed on the basis of standard SGTIN. Growing of the business units will be stacked so that to be together created with a variety of pallet units (container), which will be labelled by EPC code, created under SSCC standard. Strategic locations where the production takes place (income items), loading / unloading, selling (supply items) goods, or the place where the goods are stored shall be labelled the EPC code generated by standards-based SGLN. Marked points help to locate the exact location of goods in the warehouse or goods traced anywhere in the supply chains. 


\section{Conclusion}

In this article, we have portrayed the main areas of using services of mobile network and especially the SMS service. This service is in conjunction with the process of delivery postal items, it allows creating a fast and effective bridge between the postal operator and the recipient for providing information on the status of delivery mail. Therefore, it allows one-way but also two-way communication in order to improve the process of delivering mail and also increase comfort services in a competitive environment. And that's why we can say that use of mobile networks can be described as a key to obtaining meanings competitive advantage in postal market. At the same time, there have been described necessary standards that are used for marking the various elements in the processing chain.

Use of EPC codes in the postal service can be a variety. In combination with RFID identifiers, as the medium of the EPC code, it can be used for the marking of the transport unit. Transport units can be marked with smart labels. The added value is based on itself RFID, that allows you to capture transport units without direct optical visibility, allows you to read more labels in a short time and at greater distances, as many of the radio technologies at an operating permit busy transmitting and receiving signals more efficiently than optical scanners.

The problem with the use of EPC codes in the mail may be in the absence of headers for operators at present, because there is no header and encoding scheme for the postal sector. Post Office would have to ask for header by GS1. A preferable option now appears to be using the already existing EPC standards (header) and adding the data according to their own needs; the data must remain for the structure, which binds to the selected header. It is for consideration of weather, like the CPID automotive or aerospace industry for ADI, it is worthwhile to create a coding scheme for the postal sector of some sort (PPID Postal part identification) that would more clearly reflect the needs of the sector (shipment, related services, locating and other elements). It has been a bit difficult process for approving and setting standards for operators, but the outcome could be more effective than the application of the existing standards.

\section{Acknowledgement}

- Centrum of excellence for system and services of intelligent transport systems II (048/2009/2.1/OPVaV, Activity 1.4)

- OPV-2009/1.2/01-SORO - Systematization advance technologies a knowledge between industry sphere and university environment VEGA 2014 - Modelling diffuse knowledge in enterprises valuable chains.

- $\quad$ E!7592 AUTOEPCIS - RFID Technology in Logistic Networks of Automotive Industry.

\section{References}

1. Vaculík, J., Zelík, P. (2010) Vision of implementation of mobile technology in the postal transport process, in IPoCC - International Postal and e-Communication Conference, Institute Jana Pernera, 2010 - ISBN 978-80-8530-68-0.

2. Tengler, J.(2013) System for receiving and delivery of postal items by using RFID technology and mobile service [thesis] - University of Zilina, Faculty of Operation and Economics of Transport and Communications, Department of communications; advocates 08/26/2013.

3. Bolarin, C., Guilllamon, F., Frutos, A., Lisec, A. (2011) Assessing the Impact of Prices Fluctuation on Demand Distoretion within a Multi-echelon Supply Chain, Promet - Traffic \& Transportation, vol. 23, No. 2, pp. 131-140, 2011.

4. Beneš, F., Švub, J., Kebo, V., Štolba, M. (2013) Experiments with custom manufactured rfid antenna tags. Proceedings of the 13th International Multidisciplinary Scientific GeoConference SGEM 2013, Albena, Bulgaria; ISBN: 978-954-91818-9-0; ISSN: 1314-2704

5. Fuchsíková, P., Staša, P. and Kebo, V. (2012) RFID technology for special food moulds; Proceedings of the 13th International Carpathian Control Conference (ICCC), High Tatras, Podbanské, Slovak Republic, 2012; ISBN: 978-1-4577-1866-3; DOI: 10.1109/CarpathianCC.2012.6228636

6. Kolarovszki P., Dúbravka V. (2010) The presentation of production line and warehouse management based on RFID technology through 3D modelling and animation. In: Transport and telecommunication. - ISSN 1407-6160. - Vol. 11, No. 3 (2010), s. 26-36. 